\title{
General Limit Theorems with o-Rates and Markov Processes under Pseudo-Moment Conditions
}

\author{
P. L. Butzer and 'H. Kirschfink
}

Mit-Hilfe der Dvoretzkyschen Erweiterung der Trotter-Operatoren-Methode wird ein allgemeiner Konvergenzsatz mit $0_{-}$-Ordnung für abhängige, reellwertige Zufallsvariable, dic einer Pseudomomentenbedingung genügen, bewiesen. Dieses Ergebnis wird auf allgemeine Grenzwertsätze, einen zentralen Grenzwertsatz sowic auf ein schwaches Gesetz der großen Zahlen für Markovsche Prozesse mit diskretem Zeitparameter angewandt. Ferner werdén Pseudomómentenbedingungen diskutiert und die Materie mit Ergebnissen aus der Theorie der Wahr. scheinlichkeitsmetriken verglichen.

С помощью расширения Дворецкого метода операторов Троттера цоказывается обцая теорема сходимости с'о-порядком для независимых вещественно-значных случайных величин уповлетворяющих одному условию псевдомоментов. Әтот результат применяется к общим теоремам сходимости, к одной центральной предельной теореме и ослаблённому закону больших чисел для процессов Маркова.с дискретным временным параметром. Цалее, обсуждаются детально условия псевдомоментов и результаты сравииваются с теорией вероятностиых метрик.

Making use of the Dvoretzky extension of the Trotter-operator method, a general convergence theorem with $o$-rates for dependent, real-valued random variables satisfying a pseudo-moment condition is established. Applications are to general convergence theorem's, a centràl limit theorem as well as a weak law of lárge numbers for Markov processes with discrete time param. eter. Further, pseudo-moment conditions áre discussed in detail and the results are compared with the theory of probability-metrics.

\section{Introduction}

The fundamental limit theorems of probability theory are generally concerned with the convergence of sums of random variables towards a given limit random variable. Apart from the type of convergence, the dependency structure of the random variables in questions as well as the particular limit random variable have to be specified. This paper is devoted to limit theorems with o-rates of convergence for sums of not necessarily independent nor identically distributed random variables, thus for arbitrarily dependent, random variables. In order that the approach is rather broad, the results will be based upon a general limit theorem with rates in form of convergence in distribution (Theorem 1), thus

$$
\left|\mathrm{E}\left[f\left(T_{n}+u\right)\right]-\mathrm{E}[f(Z+u)]\right|=o_{f}\left(\varphi(n)^{r} V(n)\right) \quad(n \rightarrow \infty),
$$

for each fixed. $u \in \mathbb{R}$; here the normalized sums $T_{n}=\varphi(n) \sum_{i=1}^{n} X_{i} ;\left(X_{i}\right)_{i \in \mathbb{N}}$ being an arbitrary sequence of real, possibly dependent random variables, $\varphi: \mathbb{N} \rightarrow \mathbb{R}$ is a normalizing function with $\varphi(n)=\iota(1), n \rightarrow \infty, Z$ a $\varphi$-decomposable random variable (i.e. for each $n \in \mathbb{N}$ there exist independent random variables $Z_{i}\left(=Z_{n i}\right), 1 \leqq i \leqq n$, 
such that for the distribution $P_{Z}$ of $Z$ one has $\left.\bar{P}_{Z}=P_{\varphi(n)} \sum_{i=1}^{n} Z_{l}\right), f$ belongs to the convergence determining class $C^{r}(\mathbb{R})$ for any $r \in \mathbb{P}=\{0,1,2 ; \ldots\}$ (see (2.1) for the definition), and $V(n)$ is defined in terms of the absolute pseudo-moments of order $r$. of the random variables in question (see (2.12ii) for the definition). This general theorem will-be applied in particular to (sums of) random variables $X_{i}, i \in \mathbb{N}$, which form a non-homogeneous Markov process with a discrete timéparameter.(Theorem 2), namely to a stochastic process distinguished by the Marko property (see (4.1)).

Until 1975 most of the results known in this direction, at least in the particular case of the central limit theorem (for which the limit random variable $Z=X^{*}$, the normally distributed random variable with mean 0 and variance 1 and $\varphi(n)=\left(\sum_{i=1}^{n} \operatorname{Var}\left(X_{i}\right)\right)^{-1 / 2}$ ), dealt with not necessarily independent random variables that satisfy Doeblin's condition or the so-called $\varphi$-mixing condition. Both additional assumptions roughly mean that the random variables are "asymptotically independent"; they are rarely satisfied for Markov processes. In this respect,' for example, Lifshits [26] established under a condition upon the maximum coefficient of the correlation (related to the above assumptions [27]) the result $\sup \left|F_{n}(x)-F_{X} \cdot(x)\right|=o(1)$, $n \rightarrow \infty$, where $F_{n}(x)$ is the distribution function of $\sum_{i=1}^{n}\left(X_{i}-\operatorname{E} X_{i}\right) / \operatorname{Var}\left(\sum_{i=1}^{n} \cdot X_{i}\right)$. Then in 1976 LANDErs and Rogge [24] studied the central limit theorems for Markov chains for the convergence of the distribution function of $\left(\sum_{i=0}^{n} f\left(X_{i}(\omega)\right)-n \pi_{k}\right) / \operatorname{Var} \sqrt{n \pi_{k}}$, where $\pi_{k}$ is defined as the reciprocal of the mean recurrence time of the state $k$ and $\dot{f}=\mathbb{1}_{\{i\}}$, towards $F_{X^{\bullet}}$, obtaining the order $O\left(n^{-\alpha}\right)$ for each $\alpha<1 / 4$ under just a weak third moment condition. In this regard BolthausEn [5] established the rate $o\left(n^{-\varepsilon}\right)$ for $\varepsilon<1 / 3-1 / 6(c+1)$ and some $c \geqq 3$ for which $\mathrm{E}\left[\left|X_{i}\right|^{c}\right]<\infty$. Both papers actually deal with positive recurrent irreducible Markov chains with countable state space.

Our version of the central limit theorem for Markov processes (Theorem 3 ) gives for each fixed $u \in \mathbb{R}$ the estimate

$$
\left|\mathrm{E}\left[f\left(A_{n}=1 \sum_{i=1}^{n} X_{i}+u\right)\right]-\mathrm{E}\left[f\left(X^{*}+u\right)\right]\right|=o_{j}\left(A_{n}{ }^{-r} M(n)\right) \quad(n \rightarrow \infty)
$$

for $f \in C^{r}$, where $A_{n}=\left(\sum_{i=1}^{n} a_{i}{ }^{2}\right)^{1 / 2}$, the $a_{i}$ being positive reals and $M(n)$ as in $(2.12 \mathrm{i})$. In the particular case of Markov processes having stationary, independent increments with mean 0 (thus for identically distributed random variables) this estimate turns out to be

$$
\left|\mathrm{E}\left[f \cdot\left(n^{-1 / 2} \sum_{i=1}^{n} X_{i}+u\right)\right]-\mathbf{E}\left[f\left(X^{*}+u\right)\right]\right|=\varepsilon_{f}\left(n^{(2-r) / 2}\right) \quad(n \rightarrow \infty)
$$

for each $u \in \mathbb{R}$ with $A_{n}=n^{1 / 2}$."

Concerning the assumptions needed for our results, moment type conditions will also be required, namely the conditional pseudo-moment condition (3.3) of order $r$ as well as a pseudo-Lindeberg condition (2.11) of order $r$. Whereas the dependency structure of the random variable is unrestricted, it will depend indirectly upon moment conditions, in particular upon our pseudo-moment condition. It will in any case cover Markov processes (and martingale difference sequences) for both of which the dependency structure "depends" on the past, i.e., the random variables depend only upon their predecessors; they will be said to be dependent from below. This aspect will especially be dealt with in Section 3.2. Alternative conditions to the weak pseudo- 
moment condition (3.3) will also be examined (Section 5); it can indeed by replaced by the same non-conditional moment condition already used in BUTzER and HaHN [8], noting that the functions of $C^{\gamma}$ are bounded. In fact, (3.3) can be replaced by the condition

$$
\sum_{i=1}^{n} \mathrm{E}\left[\left|X_{i}^{j}-Z_{i}^{j}\right|\right]=c\left(\varphi(n)^{r} \sum_{i=1}^{n} \mathrm{E}\left[\left|X_{i}-Z_{i}\right|^{r}\right]\right) \quad \because(1 \leqq j \leqq r) .
$$

This will enable one to extend assertion (1.1), valid pointwise in $u \in \mathbb{R}$, to the uniform assertion (5.8) of Theorem 6.

It is rather interesting to note that results such as these may also be deduced from the well-known Kantorovitch-Rubinstein-Dudley theorem (see e.g: $[17,37]$ ), or for the analogous $M(n)$-version by a lemma and a theorem of ZoLOTAREv [36]. The Kantorovitch-Rubinstein theorem gives for the distance of two random variables the estimate

$$
\sup _{u \in \mathbb{R}}\{|\mathrm{E}[f(X+u)]-\mathbf{E}[f(Z+u)]|\} \leqq \mathrm{E}\left[|X-Z|^{\boldsymbol{a}}\right], \quad 0<s \leqq 1,
$$

where $f \in D_{s}, D_{s}$ as in (6.5). Applying it to our situation, one has for $X=T_{n}$ and $Z=Z$ the estimate (compare Theorem 8)

$$
\sup _{u \in \mathbb{R}}\left\{\left|\mathrm{E}\left[f\left(T_{n}+u\right)-f(Z+u)\right\}\right|\right\} \leqq \sum_{i=1}^{n} \varphi(n)^{8} \mathrm{E}\left[\left|X_{i}-\dot{Z}_{i}\right|^{8}\right]
$$

However, it is important that this version of (1.2) is only valid for independent random variables $X_{i}$ and $Z_{i}$. Thus our Theorem 6 can be regarded as a certain generalization of the Kantorovitch-Rubinstein-Dudley theorem to the case of dependent random variables and stochastic processes. This matter is elaborated upon in Section 6. It should be mentioned that Bergström [4] used pseudo-moment cońditions in the case of independent identically distributed random variables already in 1953. The recent papers by PaDitz [30] and Sazonov and Ulyanov [33] re-emphasize the importance of such conditions.

. Not only will limit theorems be studied for the sums $T_{n}=\sum_{i=1}^{n} \varphi(n) X_{i}$ but also for the processes $X_{n}$ themselves for $n \rightarrow \infty$, as indicated. The latter will depend upon the structure of the increments. Whereas the literature abounds with results concerning the rate of convergence of sums of random variables which are connected in $a$ Markov process, at least in the case of the central limit theorem, the results for the $X_{n}$ mostly deal with the behaviour of $n$-step transition probabilities (cf. CHUNG [16, pp. 10] or.Grman and Skorohod [19, pp. 282]) whereby in the instance of independent and.stationary increments there is a direct connection between the increments of Markoy chains and 1 -step transition probabilities (cf. Cnovg $[16$, p. 10]). It is interesting to observe that in the particular case of independent increments the present résults practically coincide with those of BuTZER and HAHN [8] of 1978 on the convergence of sums of independent random variables towards a $\dot{\phi}$-decomposable limit random variable (Section 4.3). The latter. were generalized to the case of not necessarily independent random variables which form martingale difference sequences or arrays in Butzer, HaHN and RoEckerath $[10,11]$, and to more general types of dependent random variables in BUTzer and SchuLz [13-15], as well as to arbitrary sequences for the particular case of identically distributed random variables in BUtzer and KrRschFink [12]. As far as the authors are aware, there are no comparable general limit theorems with rates in the literature. 
The type of convergence considered in this paper is more general than that in Butzer, Hahn and Roeckerath [10], or in Butzer and Schulz [13] where the dependency structure is of type of martingale difference sequences. In fact; the convergence considered in (1.1) is that for the difference

$$
\int_{\mathbb{R}} f(x+u) d F_{T_{n}}(x)-\int_{\mathbb{R}} f(x+u) d F_{z}(x)
$$

pointwise in $u \in \mathbb{R}$, and not just for the particular case $u=0$ treated in the papers mentioned above. This is even generalized to uniform convergence for $u \in \mathbb{R}$ in Section 5. Although both assertions are equivalent in case of convergence, at least in the case of independent, identically distributed random variables, this equivalence need not remain valid for convergence with rates. If the assertion (1.3) is equippèd with the rate $O\left(n^{-(r-2) / 2}\right)$ for $f \in C^{r}$, then it is equivalent to

$$
\int_{\mathbb{R}} f(x) d F_{T_{n}}(x)-\int_{\mathbb{R}} f(x) d F_{2}(x)=O\left(n^{-(r-2) / 2}\right) \quad(n \rightarrow \infty)
$$

or even to each of the two

$$
\begin{aligned}
& \int_{\mathbb{R}} x^{j} d F_{T_{n}}(x)-\int_{\mathbb{R}} x^{j} d F_{,}(x)=O\left(n^{-(r-2) / 2}\right) \quad(1 \leqq j \leqq r-1), \\
& \mathrm{E}\left[X^{i}\right]=\mathrm{E}\left[Z^{j}\right] . \quad(1 \leqq j \leqq r-1)
\end{aligned}
$$

in the independent identically distributed case (sce BUTzER and HahN [9]). However, nothing seems to be known in this respect if the random variables are not necessarily independent identically distributed or if the $\mathcal{O}\left(n^{-(r-2) / 2}\right)$-rates are replaced by o-rates, which is the situation of the present paper.

\section{Notations and preliminaries}

Let $C=C(\mathbb{R})$ denote the class of all real-valued; bounded, uniformly continuous functions defined on the reals $\mathbb{R}$, endowed with norm $\|f\|:=\sup |f(x)|$. For $r \in \mathbb{P}$ $=\mathbb{N} \cup\{0\}$ set

$$
C^{0}=C, \quad C^{r}=\left\{g \in C ; \dot{g}^{(j)} \in C, 1 \leqq j \leqq r\right\},
$$

the seminorm on $C^{r}$ being given by $|g|_{c^{r}}=\left\|g^{(r)}\right\|$.'Concerning the random variables in question, the class $3(\Omega, \mathfrak{A})$ plays an importent role. If $(\Omega, \mathfrak{A}, P)$ is a probability space, then $\mathfrak{Z}(\Omega, \mathfrak{A}):=\{X ; X$ is $\mathfrak{A}-\mathfrak{B}$-measurable $\}$, where $\mathfrak{B}$ is the Borel $\sigma$-algebra on $\mathbb{R}$. If $X \in \mathfrak{Z}(\Omega, \mathfrak{A})$, and $\mathfrak{E}$ a sub- $\sigma$-algebra of $\mathfrak{A}$, then the $\mathfrak{E}$-measurable furiction $\mathrm{E}[X \mid \mathfrak{E}]$, defined by $\int_{F} \mathrm{E}[X \mid \mathcal{E}] d P=\int_{F} X d P$ for every $F \in \mathbb{E}$; is known as the conditional expectation of $X$, given $(F$. Its properties will also be needed. Let $X, Y$ $\in \mathbb{Z}(\Omega, \mathfrak{A})$ such that $\mathrm{E}[X]<\infty, \mathrm{E}[Y]<+\infty$, and $\mathfrak{E} \subset \mathfrak{A}$. They read:

$$
\begin{aligned}
& X \geqq Y \text { a.s. implies } \mathrm{E}[X \mid \mathbb{E}] \geqq \mathrm{E}[Y \mid \mathbb{E}] \text { a.s. } \\
& X=c \text { a.s. ( } c \text { a constant) implies } \mathrm{E}[X \mid \mathbb{E}]=c \text { a.s. } \\
& \mathrm{E}[\alpha X+\beta Y \mid \mathrm{G}]=\alpha \mathrm{E}[X \mid \mathrm{E}]+\beta \mathrm{E}[Y \mid \mathbb{E}] \text { a.s. }(\alpha, \beta \in \mathbb{R}) . \\
& \mathrm{E}[\mathrm{E}[X \mid \mathbb{E}] \mid=\mathrm{E}[X] .
\end{aligned}
$$

Let $X$ be $\mathbb{E}$-measurable; then $\mathrm{E}[X \mid \mathfrak{E}]=X$ a.s: 
Let $X$ be $(F-m e a s u r a b l e$ and, $\mathrm{E}[X Y]<\infty$, then $\mathrm{E}[X Y \mid \mathcal{E}]=X \mathrm{E}[Y \mid \mathbb{E}]$ a.s.

'Let $\mathscr{U}(X)$ and $(5)$ be independent; then $\mathrm{E}[X \mid \mathfrak{F}]=\mathrm{E}[X]$ a.s.

Let $\mathfrak{X}\left(\mathfrak{A}(X), \mathfrak{E}_{1}\right)$ and $\mathfrak{E}_{2}$ be independent for two sub- $\sigma$-algebras

$\mathfrak{E}_{1}, \mathfrak{E}_{2} \stackrel{\vdots}{\doteqdot} \mathfrak{A}$. Then $\mathrm{E}[X \mid \mathfrak{E}]=\mathrm{E}\left[X \mid \mathfrak{E}_{1}\right]$ a.s. with $\mathfrak{E}=\mathscr{A}\left(\mathfrak{E}_{1}, \mathfrak{E}_{2}\right)$.

For the proofs of these properties see LAHA and Rohatgi [23, pp. 358], BaUER [3, pp. 289] or GaENSSLER and StUTe [18, pp. 185].

The following generalizations of the well-known Lindeberg condition will play an important role in the proofs.

Definition 1: The sequence $\left(X_{i}\right)_{i \in \mathbb{N}}$ of real random variables having a finite móment of order $r$, some $0<r<\infty$, is said to satisfy the generalized Lindeberg condition of order $r$ if, for every $\delta>0$,

$$
\frac{\sum_{i=1} \int_{|x| \geqq \delta / \varphi(n)}|x|^{\top} d F_{X_{i}}(x)}{\sum_{i=1}^{n} \mathrm{E}\left[\left|X_{i}\right|^{\top}\right]} \rightarrow 0 \quad(n \rightarrow \infty)
$$

The case $r=2$ with $\varphi(n)=\left(\sum_{i=1}^{n} \mathrm{E}\left[X_{i}{ }^{2}\right]\right)^{1 / 2}$ reduces to the usual Lindeberg condition (cif. Butzer, HahN and Westphal [7]).

Definition 2: Two sequences $\left(X_{i}\right)_{i \in \mathbb{N}}$ and $\left(Z_{i}\right)_{i \in \mathbb{N}}$ of real random variables with

i) finite absolute moments of order $r$ or

ii) $\mathrm{E}\left[\left|X_{i} \div Z_{i}\right|^{p}\right]<\infty$,

are. said to satisfy a generalized pseudo-Lindeberg condition of order $r$ if, for every $\delta>0$,

$$
\sum_{i=1}^{n} \int_{|x| \geqq \delta / \varphi(n)}^{\sigma}|x|^{r} d\left(F_{X_{i}}(x)-F_{Z_{i}}(x)\right)= \begin{cases}c_{\delta}(M(n)) \text { or } & (n \rightarrow \infty), \\ c_{\delta}(V(n)) & \end{cases}
$$

where

$$
\begin{aligned}
& M(n)=\sum_{i=1}^{n}\left(\mathrm{E}\left[\left|X_{i}\right|^{r}\right]+\mathrm{E}\left[\left|Z_{i}\right|^{r}\right]\right) \\
& \because \vdots \bar{n})^{*}=\sum_{i=1}^{n}\left(\mathrm{E}\left[\left|X_{i}-Z_{i}\right|^{r}\right]\right)
\end{aligned}
$$

There is the following trivial connection between the generalized Lindeberg and the generalized pseudo-Iindeberg condition (2.11i).

Lemma 1: Let $\left(X_{i}\right)_{i \in \mathbb{N}}$ and $\left(Z_{i}\right)_{i \in \mathbb{N}}$ be two sequences in $3(\Omega, \mathfrak{U})$ having finite absolute moments of order $r, 0<r<\infty$. If each of the sequences fulfils a generalized Lindeberg condition of order $r$, then both together fulfil a generalized pseudo-Lindeberg condition $(2,11$ i) of order $r$. 


\section{General limit theorems with o-rates}

The following main approximation theorem for sums of not necessarily independent randoim variables will be established by a mòdification of the Lindeberg-Trotter operator-theoretic approach as tailored to the situation of dependent random variables by means of Droretsky's tèlescoping argument. For this purpose the assumptions are the generalized pseudo-I,indeberg condition of order $r$ for the random variables $X_{i}$ and the decomposition components $Z_{i}$ as well as a conditional pseudo-moment condition of order $r$.

Theorem $1^{\prime}$ : Let $\left(X_{i}\right)_{i \in \mathbb{N}}$ be a sequence of $\mathcal{Z}(\Omega, \mathfrak{A})$ (not necessarily identically distributed nor independent) and $Z$ a $\varphi$-decomposable random variable with $\mathrm{E}[Z]^{\prime}=0$, such that

or

$$
\zeta_{r i}=\mathrm{E}\left[\left|X_{i}\right|^{r}\right]<\infty \text { and } \xi_{r i}=\mathrm{E}\left[\left|Z_{i}\right|^{r}<\infty\right. \text {, }
$$

$$
\therefore \nu_{r i}=\mathrm{E}\left[\left|X_{i}-Z_{i}\right|^{r}\right]<\infty
$$

for some $r \geqq 2$. Assume further that the sequences of random variables $\left(X_{i}\right)_{i \in \mathbb{N}}$ and de composition components $\left(Z_{i}\right)_{i \in \mathbb{N}}$ together satisfy

the generalized pseudo-Lindeberg condition (2.11 i)

or

$$
\text { the generalized pseudo-Lindeberg condition (2.11 ii) }
$$

of orders $r$, as well as

$$
\sum_{i=1}^{n} \mathbf{E}\left[\left(X_{i}^{j}-Z_{i}^{j}\right) \mid \mathfrak{A}_{n i}\right](\omega)=\left\{\begin{array}{l}
o\left(\varphi(n)^{r} M(n)\right), \text { or } \\
\cdot\left(\varphi(n)^{r} V(n)\right),
\end{array} \quad\right. \text { a.s. }
$$

where $\mathfrak{A}_{n i}=\mathfrak{U}\left(X_{1}, \ldots, X_{i-1}, Z_{i+1}, \ldots, Z_{n}\right)$.

Then for any $f \in C^{\top}$ there holds for each fixed $u \in \mathbb{R}$ the estimate

$$
\left[\mathrm{E}\left[f\left(T_{n}+u\right)\right]-\mathrm{E}[f(Z+u)]\right]=\left\{\begin{array}{l}
c_{f}\left(\varphi(n)^{r} M(n)\right) \\
c_{f}\left(\varphi(n)^{r} V(n)\right)
\end{array} \quad(n \stackrel{!}{\rightarrow} \infty) .\right.
$$

Proof: Regarding the first case, setting $R_{n i}=\sum_{k=1}^{i-1} X_{k}+\sum_{k=i+1}^{n} Z_{k}, 1 \leqq i \leqq n$, $n \in \mathbb{N}$, the telescoping argument and a double application of Taylor's formula for $f \in C^{r}$ yields for each $u^{\prime} \in \mathbb{R}$ the identity and estimate

$$
\begin{aligned}
\mid \mathrm{E} & {\left[f\left(T_{n}+u\right)\right]-\mathrm{E}[f(Z+u)] \mid } \\
= & \left|\mathrm{E}\left[f\left(\sum_{i=1}^{n} \varphi(n) X_{i}+u\right)-f\left(\sum_{i=1}^{n} \varphi(n) Z_{i}+u\right)\right]\right| \\
= & \left|\sum_{i=1}^{n} \mathrm{E}\left[f\left(\varphi(n) R_{n i}+u+\varphi(n) X_{i}\right)-f\left(\varphi(n) R_{n i}+u+\varphi(n) Z_{i}\right)\right]\right| \\
\leqq & \left|\sum_{i=1}^{n} \sum_{j=1}^{r} \frac{\varphi(n)^{j}}{j !} \mathrm{E}\left[f^{(i)}\left(\varphi(n) R_{n i}+u\right)\left(X_{i}^{j}-Z_{i}^{i}\right)\right]\right| \\
& +\sum_{i=1}^{n} \frac{1}{(r-1) !} \mid \int_{0}^{1}(1-t)^{r-1} \mathrm{E}\left[f^{(r)}\left(\varphi(n) R_{n i}+u+t \varphi(n) X_{i}\right)\left(\varphi(n) X_{i}\right)^{r}\right. \\
& \left.-f^{(r)}\left(\varphi(n) R_{n i}+u\right)\left(\varphi(n) X_{i}\right)^{r}\right] d t \mid
\end{aligned}
$$




$$
\begin{aligned}
& +\sum_{i=1}^{n} \frac{1}{(r-1) !} \mid \int_{0}^{1}(1-t)^{r-1} \mathrm{E}\left[f^{(r)}\left(\varphi(n) R_{n \mathrm{i}}+u+t \varphi(n) \dot{Z}_{\mathrm{i}}\right)\right. \\
& \left.\times\left(\varphi(n) Z_{i}\right)^{r}-f^{(r)}\left(\varphi(n) \dot{R}_{n i}+u\right)\left(\varphi(n) Z_{i}\right)^{r}\right] d t \mid
\end{aligned}
$$

To estimate the first term, namely

$$
\sum_{i=1}^{n} \sum_{j=1}^{r} \frac{\varphi(n)^{j}}{j !} \mathrm{E}\left[f^{(i)}\left(\varphi(n) R_{n i}+u\right)\left(X_{i}^{j}-Z_{i}^{j}\right)\right],
$$

one has on account of $(2.4)-(2.6)$, noting the $\mathfrak{A}_{n i}$-measurability of the $R_{n i}$, and that $\left|f^{(j)}(x)\right| \leqq N_{f}^{(j)}, x \in \mathbb{R}$, for $1 \leqq j \leqq r$ since $f \in C^{r}$, together with condition $(3.3)$, for each $u \in \mathbb{R}^{\prime}$,

$$
\begin{aligned}
& \sum_{i=1}^{n} \mathrm{E}\left[f^{(i)}\left(\varphi(n) R_{n i}+u\right)\left(X_{i}^{j}-Z_{i}^{j}\right)\right] \\
& =\sum_{i=1}^{n} \mathrm{E}\left[f^{(j)}\left(\varphi(n) R_{n i}+u\right) \mathrm{E}\left[\left(X_{i}^{i}-Z_{i}^{j}\right) \mid \mathfrak{A}_{n i}\right]\right] \\
& \leqq N_{r}^{(i)} \sum_{i=1}^{n}\left|\mathrm{E}\left[\mathrm{E}\left[\left(X_{i}^{j}-Z_{i}^{j}\right) \mid \mathfrak{X}_{n i}\right](\omega)\right]\right| \\
& =N_{j}^{(j)} \cdot\left(\varphi(n)^{r} M(n)\right) \quad \text { a.s. }
\end{aligned}
$$

Multiplýing this estimate by $\varphi(n)^{j} / j$ ! and summing over $1 \leqq j \leqq r$ yields that (3.6) is of the order $c\left(N_{f}(r, \varphi) \varphi^{\tau}(n) M(n)\right)$ with $N_{f}(r, \varphi):=\sum_{j=1}^{r} \varphi(n)^{j} N_{f}^{(j)} / j !$. Concerning the second and third terms of (3.5), let us show that they are of order $o(1)$ after being multiplied by $\varphi(n)^{-r} M(n)^{-1}$. Indeed, since $f \in C^{r}, \mid f^{(r)}\left(\varphi(n) R_{n i}+u+t \varphi(n) X_{i}\right)$ $-f^{(r)}\left(\varphi(n) R_{n \mathrm{i}}+u\right) \mid<\varepsilon$ for $\left|X_{\mathrm{i}}\right|<\delta / \varphi(n)$ since $0<t<1$. Hence

$$
\begin{aligned}
\mathrm{DE}(t):= & \mathrm{E}\left[\left|\left\{f^{(r)}\left(\varphi(n) R_{n i}+u+t \varphi(n) X_{i}\right)-f^{(r)}\left(\varphi(n) R_{n i}+u\right)\right\} X_{i}{ }^{r}\right|\right. \\
& \left.\times \cdot\left\{\mathbb{1}_{|x|<\delta / \varphi(n)}+\mathbb{1}_{|x| \geqq \delta / \varphi(n)}\right\}\right] \leqq \varepsilon \zeta_{r i}+2|f|_{c^{r}} \int_{x \geqq \delta / \varphi(n)}|x|^{r} d F_{X_{i}}(x):
\end{aligned}
$$

This gives

$$
\begin{aligned}
& \frac{1}{M(n)} \sum_{i=1}^{n} \frac{1}{(r-1) !} \int_{0}^{1}(1-t)^{r-1} \mathrm{DE}(t) d t \\
& \leqq \frac{1}{M(n)} \sum_{i=1}^{n} \frac{-1}{(r-1) !}\left\{\int_{0}^{1}(1-t)^{r-1}\left\{\varepsilon \zeta_{r i}+2|f|_{c^{r}} \int_{|x| \geqq \delta / \varphi(n)}|x|^{r} d F_{X_{i}}(x)\right\} d t\right\} \\
& =\frac{\varepsilon}{r !}+\left.2|f|\right|^{r} \frac{1}{M(n) r !} \sum_{i=1}^{n}\left\{\int_{|x| \succeq \delta / \varphi(n)}|x|^{r} d F_{X_{i}}(x)\right\}
\end{aligned}
$$

On account of the estimate of (3.6), as well as of (3.7) and its counterpart for $Z_{i}$, this yields for each $u \in \mathbb{R}$ in view of the generalized pseudo-Lindeberg condition (2.11i) 
for the random variables $X_{i}$ and $Z_{i}$,

$$
\begin{aligned}
& \frac{1}{\varphi(n)^{r} M(n)}\left\{\mathrm{E}\left[f\left(T_{n}+u\right)\right]-\mathrm{E}[f(Z+u)]\right\} \leqq c_{f}(1)+\frac{\varepsilon}{r !}+\frac{2|f|_{C r}}{r !} \\
& \times \frac{1}{M(n)} \int_{|x| \geqq \delta / \varphi(n)}|x|^{r} d\left(F^{r} x_{t}(x)-F_{Z_{l}}(x)\right)=o_{f}(1) .
\end{aligned}
$$

The proof for the second case follows similarly as above by replacing conditions (3.1 i),-(3.2i), (3.3i) by (3.1 ii), (3.2ii), (3.3ii), and $M(n)$ by $V(n)$, respectively

Remark 1: An analogous proof for the same result could also be carried out by using the random variables

$$
R_{n i}^{*}=\sum_{k=1}^{i-1} Z_{i}+\sum_{k=i+1}^{n} X_{i}, \quad \mathfrak{T}_{n i}^{*}=\mathfrak{A}\left(Z_{1}, \ldots, Z_{i-1}, X_{i-1}, \ldots, X_{n}\right)
$$

instead of the $R_{n i}, \mathfrak{A}_{n i}$ and, in place of condition (3.3i),

$$
\sum_{i=1}^{n} \mathrm{E}\left[X_{i}^{i}-Z_{i}^{j} \mid \mathfrak{A}_{n i}^{*}\right]=o\left(\varphi(n)^{\mathbf{r}} M(n)\right) \text { a.s. } \quad(n \rightarrow, \infty) .
$$

A $V(n)$-version follows analogously. Both results are due to the $\mathfrak{A}_{n i}^{*}$-measurability of the $R_{n i}^{*}$.

Corolla'ry 1 : If the random variables, $X_{i}$ as well as the decomposition components $Z_{i}, i \in \mathbb{N}$, are in addition identically distributed and $\varphi$ is such that $\varphi(n)=o(1), n \rightarrow \infty$, then under assumption (3.3i) one has for $f \in C^{r}$ and each $u \in \mathbb{R}$

$$
\left|\mathrm{E}\left[f\left(T_{n}+u\right)\right]-\mathrm{E}[f(Z+u)]\right|=\varepsilon_{f}\left(\varphi(n)^{r} n\left(\zeta_{r 1}+\xi_{r 1}\right)\right)=i_{f}\left(\frac{\varphi(n)^{r}}{n}\right) \quad(n \rightarrow \infty) .
$$

The result follows from Theorem 1 if the pseudo-Lindeberg condition for the' $X_{i}$ and $Z_{i}$ can be shówn to follow for $\varphi(n)=o(1)$. But for identically distributed random variables this condition reduces to $\int_{|x| \geqq \delta / \Phi(n)}|x|^{\dagger} d F_{X_{i}}(x) \rightarrow 0$. for each $\delta>0$, which is satisfied automatically since $\delta / \varphi(n) \stackrel{|x| \geqq \delta / \Phi(n)}{\rightarrow} \infty, n \rightarrow \infty$

Remark 2: 1. The term $\mid \mathrm{E}\left[f\left(\left[T_{n}+u\right)\right]-\mathrm{E}[f(Z)+u] \mid\right.$ in (3.4) tends to zero for $n \rightarrow \infty$ if $\varphi(n)^{r} M(n)$ (or $\varphi(n)^{r} . V(n)$ ) is bounded. In the identically distributed case this is fulfilled for $\varphi(n)=n^{-1 / r}$. 2. According to our knowledge, no results dircetly comparable to those of Theorem 1 seem to be contained in the literature. However, results are known for more particular sequences of random variables for which the dependency structure is fixed (which are also subsumed under Theorem 1). In the case of martingale difference sequences let us refer to the papers of Basu [2]. Kato [21], Prakasa Rao [31], Rychlik [32], Scott [34] and Strobel [35]. 3. The constant $2|f| c^{r} / r$ ! in the estimate (3.7) has in the case of independent random variables in regard to $O$-estimates been improved by the factor $\sum_{j=0}^{r-1} \bar{c}_{j}\left\|f^{(i)}\right\|_{C} / j !+.2 \mathrm{~L}_{f} / r !$, where $\mathrm{L}_{f}$ is the
Lipschitz constant of $f^{(r-1)}$, by ANASTASsiov [1].

Since most of the applicable structures are actually dependencies upon the past, the following differentiation is meaningful, especially for Markov processes. Part c) was formulated in the case of Banach-valued random variables in [12] in connection with Donsker's weak invariance principle.

Definition 3 : Let $\left(X_{i}\right)_{i \in \mathbb{S}}$ be a, sequence of real random variables on some probability space $(\Omega, \mathfrak{A}, P)$. It is said to be

a) dependent from below if, for each $1 \leqq i \leqq n, n \in \mathbb{N}$, $P\left(X_{i} \in B \mid X_{1}, \ldots, X_{i-1}, X_{i+1}, \ldots, X_{n}\right) \stackrel{\cdots}{=} P\left(X_{i} \in B \mid X_{1}, \ldots, X_{i-1}\right)$ a.s. $(B \in \mathfrak{B})$.

b) dependent from above if, for each $1 \leqq i \leqq n, n \in \mathbb{N}$, $P\left(X_{i} \in B \mid X_{1}, \ldots, X_{i-1}, X_{i+1}, \ldots, X_{n}\right)=P\left(X_{i} \in B \mid \dot{X}_{i+1}, \ldots, X_{n}\right)$ a.s. $(B \in \mathfrak{B})$. 
c) expectationally dependent from below or from above if, for each $1 \leqq i \leqq n, n \in \mathbb{N}$

$$
\text { I } \mathrm{E}\left[X_{i} \mid X_{1}, \ldots, X_{i-1}, X_{i+1}, \ldots, X_{n}\right]=\left\{\begin{array}{ll}
\mathrm{E}\left[X_{i} \mid X_{1}, \ldots, X_{i-1}\right] \\
\mathrm{E}\left[X_{i} \mid X_{i+1}, \ldots, X_{n}\right] .
\end{array}\right. \text { a.s. }
$$

Examples for random variables that are expectationally dependent from below are martingale difference sequences (seé [12]) and Markov processes (see below); dependent from above are inverse martingale difference sequences and inverse Markor chains.

Lemma 2: Examination of condition (3.3) in the light of Definition 3 leads to the following statements:

a) If $X$ is any random variable, $\mathfrak{E}$, $\mathfrak{F}$ are two sub- $\sigma$-algebras of $\mathfrak{A}$, then $P(X \in B \mid \mathfrak{E})$ $=P(X \in B \mid \mathfrak{F})$ for all $B \in \mathfrak{B}$ implies $\mathrm{E}[X \mid \mathfrak{F}]=\mathrm{E}[X \mid \mathfrak{F}]$ a.s.

b) If $\left(X_{i}\right)_{i \in \mathbb{N}}$ is a sequence of random variables that is dependent from below, then it is expectationally dependent from below.

c) If $\left(X_{\mathrm{i}}\right)_{i \in \mathbb{N}}$ is dependent from above, then it is expectationally so.

Proof: The hypothesis gives in particular for $B=(-\infty, t]$ that $P(X \leqq t \mid E)$ $=P(X \leqq t \mid \mathfrak{F})$, so that $F_{X}(t \mid \mathbb{E})=F_{X}(t \mid \mathfrak{F})$. This yields $\mathrm{E}[X \mid \mathfrak{F}]=\int_{0} x d F_{X}(x \mid \mathfrak{F})$ $=\mathrm{E}[X \mid \mathfrak{F}]$, which completes the proof of part a). Parts b) and c) follow directly from a)

Lemma 3: If the sequence $\left(X_{i}\right)_{i \in \mathbb{N}}$ is expectationally dependent from below such that, for $1 \leqq j \leqq r, n \rightarrow \infty$,

$$
\sum_{i=1}^{n-} \mathrm{E}\left[\left(X_{i}^{j}-Z_{i}^{j}\right) \mid \mathfrak{E}_{i-1}\right]=\left\{\begin{array}{l}
\mathfrak{d}\left(\varphi(n)^{r} M(n)\right), \\
\mathfrak{c}\left(\varphi(n)^{r} V(n)\right),
\end{array} \quad \text { or } \quad\right. \text { a.s. }
$$

where $\mathfrak{E}_{i-1}=\mathfrak{U}\left(X_{1}, \ldots, X_{i-1}\right)$, and $Z_{i}$ are the decomposition components of Theorem 1 , then condition. $(3.3 \mathrm{i})$ or (3.3ii) is satisfied.

Proof: By Lemma $2 \mathrm{~b}$, and the independence of the $Z_{\mathfrak{i}}$ from $\mathfrak{A}_{n i}$, one has with (2.4), (2.8) and (2.9),

$$
\begin{aligned}
& \sum_{i=1}^{n} \mathrm{E}\left[\left(\dot{X}_{i}^{j}-Z_{i}^{j}\right) \mid \mathfrak{A}_{n i}\right] \doteq \sum_{i=1}^{n}\left(\mathrm{E}\left[X_{i}^{j} \mid \mathfrak{A}_{n i}\right]-\mathrm{E}\left[Z_{i}^{j} \mid \mathfrak{A}_{n i}\right]\right) \\
& =\sum_{i=1}^{n}\left(\mathrm{E}\left[X_{i}^{j} \mid \mathfrak{E}_{i-1}\right]-\mathrm{E}\left[Z_{i}^{j}\right]\right)=\sum_{i=1}^{n}\left(\mathrm{E}\left[\left(X_{i}^{j}-Z_{i}^{j}\right) \mid \mathfrak{E}_{i-1}\right]\right)
\end{aligned}
$$

Remark 3: 1. On account of Remark 1 one can also formulate both versions of Lemma 3 for sequences of random variables that are expectationally dependent from above, employing condition $(3.3)^{*}$ in place of (3.3). 2. The conditions "dependent from below" or "dependent from above" are rather severe restrictions upon dependence. For a sequence $\left(X_{i}\right)_{i \in \mathbb{S}}$ that is dependent from below it means that for each $X_{n}$ the past is not influenced by the future. In this sense dependence from below may be more restrictive than (generul) Markov dependence. In the next section, where only a special Markov-property is allowed, Markov-dependence is an exámple of dependence from below.

\section{4.' General limit theorems for Markov processes}

A Markov process with discrete time parameter is a sequence of random variables $\left(X_{i}\right)_{i \in \mathbb{D}}$ on some probability space $(\Omega, \mathfrak{A}, P)$, where each random variable $X_{i}$ is only : restricted by the Markov property

$$
P\left(X_{i} \in B \mid X_{1}, \ldots, X_{i-1}\right)=P\left(X_{i} \in B \mid X_{i-1}\right) \quad(B \in \mathfrak{B} ; i \geqq 2) .
$$


It is obvious that such a Markov process is dependent from below according to Definition 1. A Markov process with (possibly) dependent increments is a process $\left(X_{i}\right)_{i \in \mathbb{N}}$ for which the sequence of increments $Y_{i}$ with

$$
Y_{i}:=\dot{X}_{i}-X_{i-1}^{j}, \quad \dot{X}_{0}^{\prime}=0^{-} \quad \text { a.s. }
$$

is (possiblý) dependent. Whereas for a Markov process the increments are not necessarily dependent, they are nevertheless expectationally so as is seen by the following

Lemma 4: If $\left(X_{\mathrm{i}}\right)_{i \in \mathbb{N}}$ is a Markov process, then, the sequence of increments $\left(Y_{i}\right)_{i \in \mathbb{N}}$ is expectationally dependent from below.'

In fact, $\left(X_{i}\right)$ being a Markov process,

$$
\begin{aligned}
& \mathrm{E}\left[Y_{i} \mid Y_{1}, \ldots, Y_{i-1}, Y_{i+1}, \ldots, Y_{n}\right]=\mathrm{E}\left[X_{i} \mid Y_{1}, \ldots, Y_{i-1}, Y_{i+1}, \ldots, Y_{n}\right] \\
& -\mathrm{E}\left[X_{i-1} \mid Y_{1}, \ldots, Y_{i-1}, Y_{i+1}, \ldots, Y_{n}\right]=\mathrm{E}\left[X_{i} \mid Y_{i-1}\right]-\mathrm{E}\left[X_{i-1} \mid Y_{i-2}, Y_{i-1}\right] \\
& =\mathrm{E}\left[\left(X_{i}-X_{i-1}\right) \mid Y_{i-2}, Y_{i-1}\right]=\mathrm{E}\left[Y_{i} \mid Y_{i-2}, Y_{i-1}\right] .
\end{aligned}
$$

In particular, for a Markov process with independent increments the $Y_{i}$ are independent (see below).

'The following limit theorems with rates will be formulated for three instances, namely for sums of Markovian dependent random variables, for Markov processes with dependent as well as with independent increments.

\subsection{General limit theorem and central limit theorem}

At first to the general result.

Theorem 2: Let $\left(X_{i}\right)_{i \in \mathbb{N}}$ be Markov-dependent and $Z$ be a $p$-decomposable random variable with $\mathrm{E}[Z]=0$ such that $(3.1 \mathrm{i})$ or $(3.1 \mathrm{ii})$ hold for $i \in \mathbb{N}$ and $r \geqq 2$. If the sequences $\left(X_{i}\right)_{i \in \mathbb{N}}$ and $\left(Z_{i}\right)_{i \in \mathbb{N}}$ together satisfy the generalized pseudo-Lindeberg condition (2.11 i). or (2.11 ii) of order $r$, as well as condition (3.12i) or (3.12ii), then any $f \in C^{r}$ again implies the estimate (3.4i) or (3.4ii).

The proof follows immediately from Theorem 1 and Lemma 3

Now to a handy, version of the central limit theorem for Markov processes. Here we will apply. Theorem 2 to a concrete limiting random variable $Z$, namely to $X^{*}$.

Theorem 3 : Let $\left(X_{i}\right)_{i \in \mathbb{N}}$ be a sequence of random variables which form a Markov process. such that $\mathrm{E}\left[\left|X_{i}\right|^{r}\right]<\infty, i \in \mathbb{N}$, for any $r \geq 2$ as well as satisfy a generalized Lindeberg condition (2.10), of order $r$. Assume further that $\left(a_{i}\right)_{i \in \mathbb{N}}$ is any sequence of positive reals wich satisfies a Feller-type condition

$$
\lim _{n \rightarrow \infty} \max _{1 \leqq i \leqq n} \frac{a_{i}}{A_{n}}=0 \text { with } A_{n}=\left(\sum_{i=1}^{n} a_{i}^{2}\right)^{1 / 2} .
$$

If $\mathrm{E}\left[\left|Z_{i}\right|^{r}\right]<\infty$ as well as (3.12i) holds with $P_{Z_{\mathfrak{\imath}}}=P_{a_{1} X^{*}}$, then $f \in C^{r}$ yields for each fixed $u \in \mathbb{R}$

$$
{ }_{0}\left|\mathrm{E}\left[f\left(A_{n}{ }^{-1} \sum_{i=1}^{n} X_{i}+u\right)\right]-\mathrm{E}\left[f\left(X^{*}+u\right)\right]\right|=c_{f}\left(A_{n}{ }^{-r} M(n)\right) \quad(n \rightarrow \infty)
$$

Concerning the proof, $X^{*}$ is $\varphi$-decomposable for each $n \in \mathbb{N}$ into $n$ independent . normally distributed random variables $Z_{i}$ with $P_{Z_{i}}=P_{a_{i}} X^{*}$ since

$$
P_{X}=P_{A_{n}-1} \sum_{i=1}^{n} Z_{i} \text { with } \varphi(n)=A_{n}^{-1}
$$

(see [14]). Further, (4.3) in connection with Lemma 1 yields the generalized pseudoLindeberg condition (2.11i). So Theorem 2 may be applied 
Remark 4: There exists a further version of Theorem 3 in case the finiteness of the $r$-th absolute moments of the $X_{i}$ and $Z_{i}$ is replaced by the finiteness of the $r$-th-pseudo-moments of $\left(X_{i}-Z_{i}\right)$, and the generalized Lindeberg condition for $X_{i}$ together with the Feller condition for $a_{i}$ is replaced by

$$
\frac{1}{V^{\prime}(n)} \sum_{i=1}^{n} \int_{|x| \geqq \delta / \varphi(n)}|x|^{r} d\left(F_{X,}(x)-F_{a_{i} X}(x)\right)=o_{\delta}(1)
$$

for each $\delta>0$, where $V^{\prime}(n):=\sum_{i=1}^{n} \mathrm{E}\left[\left|X_{i}-a_{i} X^{*}\right|^{\gamma}\right]$ : If moreover $(3.12 \mathrm{ii})$ takes the place of (3.12i) with $V(n)$ replaced by $V^{\prime}(n)$, then assertion (4.4) reads

$$
\left|\mathrm{E}\left[f\left(A_{n}^{-1} \sum_{i=1}^{n} X_{i}+u\right)\right]-\mathrm{E}\left[/\left(X^{*}+u\right)\right]\right|=\varepsilon_{0}\left(A_{n}^{-\mathrm{r}} V^{\prime}(n)\right) \quad(n \rightarrow \infty)
$$

Corollary 2: a) If, in addition to the assumptions of Theorems 3, the $X_{i}$ are iden-' tically distributed, then for $f \in C^{r}$, and each $u \in \mathbb{R}$

$$
\left|\mathrm{E}\left[f \cdot\left(A_{n}{ }^{-1} \sum_{i=1}^{n} X_{i}+u\right)\right]-\mathrm{E}\left[f\left(X^{*}+u\right)\right]\right|=c_{f}\left(n A_{n}{ }^{-i}\right) \quad(n \rightarrow \infty) .
$$

b) If further $a_{i}:=\operatorname{Var} X_{i}, 1 \leqq i \leqq n$, then for $\dot{f} \in C^{\prime}$ and $u \in \dot{\mathbb{R}}$

$$
\left|\mathrm{E}\left[f\left(\left(\sum_{i=1}^{n} \operatorname{Var} X_{i}\right)^{-1 / 2} \sum_{i=1}^{n} X_{i}+u\right)\right]-\mathrm{E}\left[f\left(X^{*}+\dot{+} u\right)\right]\right|=s_{f}\left(n^{(2-r) / 2}\right) \text {. }
$$

c) If in particular Var $X_{i}=1,1 \leqq i<n$, then for $f \in C^{2}$ and $u \in \mathbb{R}$

$$
\left|\mathrm{E}\left[f\left(n^{-1 / 2} \sum_{i=1}^{n} X_{i}+u\right)\right]-\mathrm{E}\left[f\left(X^{*}+u\right)\right]\right|=c_{f}(1) . \quad(n \rightarrow \infty) \text {. }
$$

Apart from the papers $[5,24,26]$ mentioned in the introduction, there exist many further ones dealing with the central limit theorem for Markovian dependent random variables. Generally homogeneous Markov chäins are studiéd. Thus NaGa Ev [28, 29] considered convergence in regard to the central limit theorem for chains, comparable to Corollary $2 \mathrm{c}$ ) with 0 -rates. Additional papers in this respect a re BoLTha usen [6], Gudynas [20] and Lifshits [25].

\subsection{Processes with dependent increments}

This subsection is devoted to the behaviour of the process $\varphi(n) X_{n}=\sum_{i=1}^{n} \varphi(n) Y_{i}$
for $n \rightarrow \infty$, the increments $Y_{i}$ being assumed to be dependent.

Theorem 4: Let $\left(X_{i}\right)_{i \in \mathbb{N}}$ be a Markov process with dependent increments $\left(Y_{i}\right)_{i \in \mathbb{N}}$ as in (4.2) with $X_{0}:=0$ a.s. Let. $Z$ be a $\varphi$-decomposable random variable (with respect to $\left.\left(X_{i}\right)_{i \in \mathbb{N}}\right)$ such that $\mathrm{E}[Z]=0$ and $v_{r i}^{*}:=\mathrm{E}\left[\left|Y_{i} \perp Z_{i}\right|^{*}\right]<\infty, r \geqq 2$. If the sequences $\left(Y_{i}\right)_{i \in \mathbb{N}}$ and $\left(Z_{i}\right)_{i \in \mathbb{N}}$ together satisfy a pseudo-Lindeberg condition (2.11 ii) of order $r$ as well as

$$
\sum_{i=1}^{n} \mathrm{E}\left[\left(Y_{i}^{j}-Z_{i}^{j}\right) 、 \mid \mathfrak{S}_{i-1}^{*}\right]=o\left(\varphi(n)^{r} V^{*}(n)\right) \quad(1 \leqq j \leqq r ; n \rightarrow \infty) \text { a.s. }
$$

with $\mathbb{E}_{i-1}^{*}:=\mathfrak{A}\left(Y_{1}, \ldots, Y_{i-1}^{i}\right)$ and $V^{*}(n):=\sum_{i=1}^{n} \nu_{r i}^{*}$, then for $f \in C^{r}$ and each $u \in \mathbb{R}$

$$
\left|E\left[f\left(\varphi(n) X_{n}+\dot{u}\right)\right]-\mathrm{E}[f(Z+u)]\right|=\varepsilon_{f}\left(\varphi(n)^{r} V^{*}(n)\right) \quad(\dot{n} \rightarrow \infty) .
$$

The proof follows directly from Theorem 1 and Lemmata 2 and 3 


\subsection{Processes with independent increments}

If in the results of Section 4.1 the increments $Y_{i}=X_{i}-X_{i-1}$ are assumed to be independent, which is often the situation in applications (e.g. queueing theory and simulation), then our problem reduces to a study of the rate of convergence for sums of independent random variables. In this sense Markovian dependency is a generalization of independency, as CHONG $[16$, p. 10] remarks. Now it is quite surprising that the results obtained by BUTZER and $\mathrm{HAHN}_{\text {[ }}$ [8] for sums of independent random variables (by means of Trotter operator-theoretic arguments) resemble those of Theorem 4 both in regard to the hypotheses and conclusion; there one managed to get along with a pseudo-moment condition instead of a conditional condition of this type and the convergence was uniform in $u \in \mathbb{R}$. In this situation one has the following result; it is comparable to [8, 'Theorem 12].

Theorem 5: Let $\left(X_{i}\right)_{i \in \mathbb{N}}$ be a Markov process with independent increments $Y_{i}$. Then the conclusions of Theorem 4 even remain valid if the condilional pseudomoment condition (4.5) is replaced by

$$
\sum_{i=1}^{n} \mathrm{E}\left[\left(Y_{i}^{j}-Z_{i}^{j}\right)\right]=c\left(\varphi(n)^{\dagger} V^{*}(n)\right) \quad(i \rightarrow \infty)
$$

the others remaining unchanged.

In fact, (4.5) reduces to $\left(4.5^{*}\right)$ in case the $Y_{i}$ are independent in view of $(2.8)$

\section{Pseudo-moments and genéralizations}

If one looks at the proof of 'Theorem 1 more closely one sees that the main problem is the estimation of the first term (3.6), thus to show that the double sum of (3.6) is in some way or other of the maximal order $o f\left(\varphi(n)^{r} M(n)\right)$ or $e_{f}\left(\varphi(n)^{r} V \cdot(n)\right)$. This was achieved there by employing, condition (3.3i) or (3.3 $\mathrm{ii})$ together with properties of conditional expectations in connection with the admissible dependency and measurability properties. In order to simplify this proof, thus to estimate (3.6) with the desired order, it obviously suffices to assume the difficult looking condition

$$
\begin{aligned}
& \sum_{i=1}^{n}\left|\mathrm{E}\left[f^{(j)}\left(\varphi(n) R_{n i}+u\right)\left(X_{i}^{j}-Z_{n i}^{j}\right)\right]\right|=c_{f}\left(\varphi(n)^{r} M(n)\right) \\
& \left(u \in \mathbb{R} ; 1 \leqq j \leqq r_{i} ; \dot{n} \rightarrow \infty\right),
\end{aligned}
$$

the $V(n)$-version being analogous. This is an implicit pseudo-moment condition in the sense that there is a "weighted" difference of the random variables $X_{i}^{j}$ and $Z_{n i}^{j}$. Now many estimates of pseudo-moments are known in the literature. Let us first define some types of pseudo-moments and consider their properties (compare ZoLOTAREv [37]).

Definition 4: Iet $X, Z$ be two random variables. The pseudo-moment $\nu(X, Z)$ is defined by $\nu(X, Z)=|\mathrm{E}[X-Z]|$, and the conditional pseudo-moment $\tau(X, Z ;$ (S) by $\tau(X, Z ; \mathfrak{B})=|\mathrm{E}[(X-Z) \mid(\mathfrak{S})]|$ where $\mathfrak{B}$ is a sub- $\sigma$-algebra of $\mathfrak{A}$.

. I. em ma 5 : For $c \in \mathbb{R}$ there hold

i) $v(c X, c \cdot Z) \leqq|c| \nu(X, Z)$,

ii) $v(X, Z)=\mathrm{E}[\tau(X, Z$; $(S)]$, 
iii) $\tau(c \cdot X, c \cdot Z$; (B) $) \leqq|c| \tau(X, Z$; (B) ,

iv) $\tau\left(c_{1} X, c_{1} Z ;(\mathfrak{H}) \leqq \tau\left(c_{2} X, c_{2} Z ;\right.\right.$ (S) $), \quad c_{1} \leqq c_{2}$.

Proof: We have i) $|\mathrm{E}[c \cdot X-c \cdot Z]| \leqq|c| \mathrm{E}[|X-Z|]$ and ii) $|\mathrm{E}[X-Z]|$ $=\mid \mathrm{E}[\mathrm{E}[(X-Z) \mid(3)] \mid=\mathrm{E}[\tau(X, Z$; (S) $]$; iii) follows as in i); iv) follows from $\left|\mathrm{E}\left[\left(c_{1} X-c_{1} Z\right) . \mid(B)\right]\right|=\left|c_{1} \mathrm{E}[(X-Z) \mid(B)]\right| \leqq \mid c_{2} \mathrm{E}[(X-Z) \mid$ (B) $] \mid \stackrel{r}{=} \tau\left(c_{2} X, c_{2} Z ;\right.$ (S)

In this terminology condition (5.1) reads (each $u \in \mathbb{R}$ )

$$
\sum_{i=1}^{n} \cdot v\left(f^{(j)}\left(\varphi(n) R_{n i}+u\right) X_{i}^{j} \cdot f^{(i)}\left(\varphi(n) R_{n i}+n\right) Z_{n i}^{j}\right)=c_{f}\left(\varphi(n)^{r} M(n)\right):
$$

Let us now give some conditions which are sufficient for condition (5.2) to hold, and so suffice for the proof of Theorem 1.

Lemma 6: Let $f \in C^{r}, M(n):=M(n, r)=\sum_{i=1}^{n}\left\{\mathrm{E}\left[\left|X_{i}\right|^{r}\right]+\mathrm{E}\left[\left|Z_{n i}\right|^{r}\right]\right\}$. There hold:

i) If for the pseudo-moment

thei

$$
v\left(X_{i}^{j}, Z_{n i}^{j}\right)=\cdot\left(n^{-1} \varphi(n)^{r} M(n)\right) \quad(n \rightarrow \infty)
$$

$$
\sum_{i=1}^{n} v\left(X_{i}^{j}, Z_{n i}^{j}\right)=e\left(\varphi(n)^{\uparrow} M(n)\right) .
$$

ii) There exists a constant $N_{f}^{(j)}, 1 \leqq j \leqq r$, such that

$$
\sum_{i=1}^{n} v\left(f^{(j)}\left(\varphi(n) R_{n i}+u\right) X_{i}^{j}, f^{(j)}\left(\varphi(n) R_{n i}+u\right) Z_{n i}^{j}\right) \leqq\left|N_{f}^{(j)}\right| \sum_{i=1}^{n} \nu\left(X_{i}^{j}, Z_{n i}^{j}\right) .
$$

iii) $\quad \nu\left(f^{(j)}\left(\varphi(n) R_{n i}+u\right) X_{i}^{j}, f^{(j)}\left(\varphi(n) R_{n i}+u\right) Z_{n i}^{j}\right)$

$$
=\mathrm{E}\left[f^{(j)}\left(\varphi(n) R_{n i}+u\right) \tau\left(X_{i}^{j}, Z_{n i}^{j} ; \mathfrak{A}_{n i}\right)\right] \text {. }
$$

iv) There exists a constant $N_{j}^{(j)}, 1 \leqq j \leqq r$, with

$$
\begin{aligned}
& \sum_{i=1}^{n} \mathrm{E}\left[i\left(f^{(j)}\left(\varphi(n) R_{n i}+u\right) X_{i}^{j}, f^{(i)}\left(\varphi(n) R_{n i}+u\right) Z_{n i}^{j} ; \mathfrak{A}_{n i}\right)\right] \\
& \leqq \\
& \sum_{i=1}^{n}\left|N_{f}^{(j)}\right| \nu\left(X_{i}^{j}, Z_{n i}^{(j)}\right) .
\end{aligned}
$$

v) If for the conditional pseudo-moment

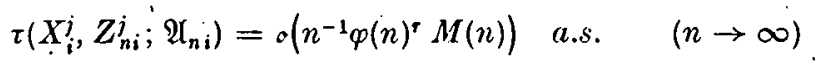

then (recall condition (3.3i) of Theorem 1)

$$
\sum_{i=1}^{n} \tau\left(X_{i}^{j}, Z_{n i}^{j} ; \mathfrak{A}_{n i}\right)=\mathfrak{e}\left(\varphi(n)^{\uparrow} M(n)\right) \quad \text { a.s. }
$$

vi) If (5.5) holds, so does (5.3), and if (5.6) holds, so does (5.4).

Proof: i) We have

$\sum_{i=1}^{n} v\left(X_{i}^{\prime}, Z_{n i}^{j}\right)=\sum_{i=1}^{n} c\left(n^{-1} \varphi(n)^{r} \cdot M(n)\right)=o\left(\varphi(n)^{r} M(n)\right)$ 
ii) Since $f \in C^{\varphi}, f^{(j)}(x)$ is bounded by a constant $N_{j}^{(j)}$, and there holds

$$
\begin{aligned}
& \sum_{i=1}^{n} v\left(f^{(j)}\left(\varphi(n) R_{n i}+u\right) X_{i}^{j}, f^{(j)}\left(\varphi(n) R_{n i}+u\right) Z_{n i}^{j}\right) \\
& \leqq \sum_{i=1}^{n} \nu\left(N_{f}^{(j)} X_{i}^{j}, N_{f}^{(j)} Z_{n i}^{j}\right) \leqq\left|N_{f}^{(j)}\right| \sum_{i=1}^{n} \nu\left(X_{i}^{j}, Z_{n i}^{j}\right)
\end{aligned}
$$

iii) There holds with Lemma $5 \mathrm{ii}$ ) and (2.7) that

$$
\begin{aligned}
& v\left(f^{(j)} \varphi\left(\varphi(n) R_{n i}+u\right) X_{i}^{j}, f^{(j)}\left(\varphi(n) R_{n i}+u\right) Z_{n i}^{j}\right) \\
& =\mathrm{E}\left[\tau\left(f^{(j)}\left(\varphi(n) R_{n i}+u\right) X_{i}^{j}, f^{(j)}\left(\varphi(n) R_{n i}+u\right) Z_{n i}^{j} ; \mathfrak{A}_{n i}\right)\right] \\
& =\mathrm{E}\left[f^{(j)}\left(\varphi(n) R_{n i}+u\right) \tau\left(X_{i}^{j}, Z_{n i}^{j} ; \mathfrak{A}_{n i}\right)\right] .
\end{aligned}
$$

iv) follows' as does ii); v) follows as i); vi) follows by Lemma 5 ii) and (2.3)

- Lemma 6 could obviously be formulated also for the $V(n)$-case; whereas Lemma 6 refers to Theorem 1 , versions attached to Theorems 3 and 4 are also possible. So one can see that conditions (5.2), (5.4), (5.5) and (5.6) suffice for (5.2) or (5.1), and so one can formulate a weaker version of Theorem 1 . The weakest alternative condition to (3.3) (or (5.1)) is condition (5.4), and will now be employed.

Theorem 6: Under the assumptions of Theorem 1 there holds, in case condition (3.3) is replaced by

$$
\sum_{i=1}^{n} \nu\left(X_{i}^{j}, Z_{n i}^{j}\right)=\left\{\begin{array}{lll}
\cdot\left(\frac{\varphi(n)^{r}}{(r-1) !} M(n)\right) & \text { or } & \\
\cdot\left(\frac{\varphi(n)^{r}}{(r-1) !} V_{i}(n)\right), & \vdots &
\end{array}\right.
$$

for each $f \in C^{r}$ the estimate

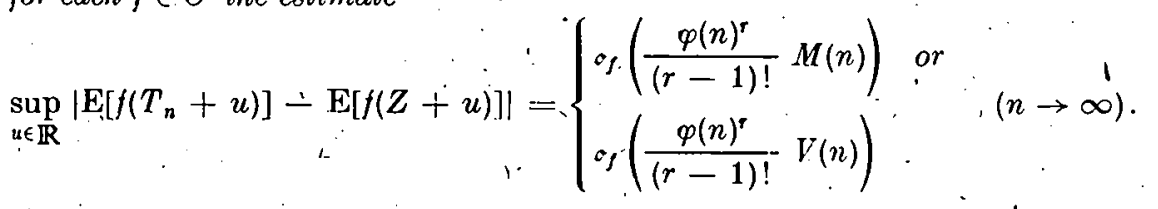

Proof: Checking the proof of Theorem 1, one just needs to re-examine the estimate of the first term of (3.5), namely (3.6). But it follows by Lemma $6 \mathrm{ii}$ ) and condition (5.7) that (3.6) is of order $c\left(\varphi(n)^{r} M(n)\right)\left(c\left(\varphi(n)^{r} V(n)\right)\right)$ uniformly for all $u \in \mathbb{R}$, as desired. So the proof is complete

Remark $5: 1$. In the particular case' of independent random variables ' $X_{i}$ Theorem. 6 coincides, with Theorem 12 in [8] so that the former is a true generalization of our earlier results for the independent case. Pseudo-moment conditions are again of decisive importance. 2 . As the proof of Theorem 6 reveals, all of the estimates derived in Sections 3 and 4 are valid not only for each individual $u \in \mathbb{R}$ but uniformly in $u \in \mathbb{R}$ provided condition (5.4) of Lemma 6 would be employed throughout. 3. Sazonov and ULYanov [33] established an 0 -estimate for the rate in the multidimensional central limit theorem for independent identically distributed random variables also in terms of pseudo-moments by using a Taylor expansion for functions $f \in C^{3}\left(\mathbb{R}^{k}\right)$.

\section{Probability metries; compàrisons with known results}

The exploitation of pseudo-moments in the case of limit theorems in probability theory is part of the theory of probability metrics (see e.g. $[17,38]$ ). In this section we will give a short survey in connection with different probability metrics. 
Definition 5: The distance $\mu$ of two random variables $X, Y$, with $\mu: \mathcal{B}(\Omega, \mathfrak{A})$ $\times \mathfrak{Z}(\Omega, \mathfrak{A}) \rightarrow[0, \infty]$, is called a probability metric if

i) $P(X=Y)=1$ implies $\mu(X, Y)=0$,

ii) $\mu(X, Y)=\mu(Y, X)$;

iii) $\mu(X, Y) \leqq \mu(X, Z)+\mu(Y ; Z)$ for a random variable $Z \in \mathcal{Z}(\Omega, \mathfrak{A})$.

Le $\mathrm{m}$ m a $7:$ In regard to the above definition one has:

a) The pseudo-moment $v$ is a probability metric.

b) The conditional pseudo-moment $\tau$ is a pseudo-probability metric, i.e., conditions ii) and iii) of Definition 5 are fulfilled, but condition i) holds only a.s.

Proof : Concerning part a), the proof is evident. Concerning b), we have:

i) $P(X=Y)=1_{1} \Rightarrow \tau\left(X, Y ;(S)=\left|\int x d\left(F_{X \mid \mathcal{G}}-F_{Y \mid \Theta}\right)(x)\right|=0\right.$ a.s. since $F_{X \mid \Theta}$ $=F_{Y \mid \Theta}$ a.s. ;

ii) evident, since $\left|\int x d\left(F_{X \mid \Theta}-F_{Y \mid \Theta}\right)(x)\right|=\left|\int x d\left(F_{Y \mid \Theta}-F_{X \mid \Theta}\right)(\dot{x})\right|$;

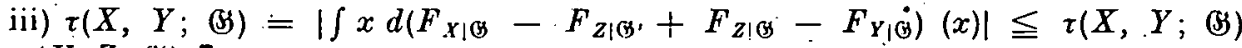
十 $\tau(Y, Z ;$ (H)

In the following, we will establish two results to be compared with Theorem 6 ; they will be deduced by well-known results of other authors.

Definition 6 : Let $X, Y \in 3(\Omega, \mathfrak{A})$. The law of $X, L(X)$, is defined by

$$
L(X)=\left\{Y \in 3(\Omega, \mathfrak{A})^{\prime} ; P_{X}^{\prime}=P_{Y}\right\}
$$

If $d$ is a classical metric, then $\gamma(X, Y ; d):=\mathbf{E}[d(X, Y)]$ defines a probability metric; the so-called minimal metric with respect to $\gamma$ is defined by

$$
\begin{aligned}
& \hat{\gamma}(X, Y ; d)=\inf \{\gamma(X, Y ; d) ; L(X, Y) \in \mathfrak{Z}(\Omega, \mathfrak{A}) \times 3(\Omega, \mathfrak{A}), \\
& \left.\dot{L}(X)=P_{X} \text { and } L(Y)=P_{Y}\right\} .
\end{aligned}
$$

In the particular case $d(x, y)=|x-y|^{\delta}, s>0, \hat{\gamma}$ is called a Wasserstein metric, and is denoted by $W_{s}\left(P_{X}, P_{Y}\right)$. The metric $\xi$ is defined by

$$
\xi(X, Y ; F)=\sup _{\mathcal{S} F}\left\{\left|\int f(x) d\left(P_{X}-P_{Y}\right)(x)\right|\right\},
$$

where $F$ is any function-class. A particular version of $\xi$ is given by

where

$$
\xi_{s}(X, Y)=\xi\left(X, Y ; D_{s}\right)
$$

$$
D_{s}= \begin{cases}f \in C, & f \in C \cap \operatorname{Lip} s\}, \\ \left.f^{(r)} \in C \cap \operatorname{Lip} \alpha\right\}, s=r+\alpha, r \in \mathbb{N}, \alpha \in(0,1], & s>1,\end{cases}
$$

and (see e.g. ' $[38])$ Lip $\alpha:=\left\{f \in C ;|f(x)-f(y)| \leqq|x-y|^{a}\right\}$.

Under these notations, the Kantorovitch-Rubinstein-Dudley Theorem reads (see e.g. $[37])$

Theorem 7: For any measurable metric $d$ and any $X, Y \in 3(\Omega, \mathfrak{X})$; there holds $\gamma(X, Y ; d)=\xi(X, Y ; G(d))$, where $G(d)=\{f \in C ;|f(x)-f(y)| \leqq d(x, y)\}$.

For the application of Theorem 7 to our results, another property of metrics is important. 
Definition 7: A metric $\mu$ is called $i d e a l$ of order $s>0$ if for any random variables $X, Y, Z \in Z(\Omega, \mathfrak{A})$ and any constant $c \in \mathbb{R}, c \neq 0$

i) $\mu(X+Z, Y+Z) \leqq \mu(X, Y)$,

ii) $\mu(c \cdot X, c \cdot Y) \leqq|c|^{8} \mu(X, Y)$,

where $Z$ is independent of $\grave{X}$ and $Y$.

In this respect there holds (see [36, Lemma 3])

Lem ma 8: For any $s>0, \xi_{s}$ is an ideal metric of order $s$ in the maximal subset of $3(\Omega, \mathfrak{A})$ within which the values of $\xi_{s}$ are finite.

Since for the Kantorovitch-Rubinstein theorem the function class $G(d)$ is needed, and since the metric $\xi$ is ideal only for the particular function class $D_{s}, s>0$, for our application our function class has to fulfil both conditions. This means that $D_{s} \cap G(d)$ $=D_{s}, 0<s \leq 1$. So our application of the Kantorovitch-Rubinstein theorem is only valid: for the metric $\xi$ with $0<s \leqq 1$.

Theorem 8: Let $X, Y \in \mathcal{B}(\Omega, \mathfrak{X})$ and $f \in D_{s}, 0<s \leqq 1$. Then' .

$$
\sup _{u \in \mathbb{R}}\{|\mathrm{E}[\dot{f}(X+u)-f(Y+u)]|\} \leqq \mathrm{E}\left[|\dot{X}-\dot{Y}|^{\bullet}\right] \text {. }
$$

Proof: One has by Lemma 8 and Theorem 7 that the left-hand side of $(6.1)$ is estimated by

$$
\begin{aligned}
& \sup _{f \in D_{s}, u \in \mathbb{R}}\{|\mathrm{E}[f(X+u)]-\mathrm{E}[f(Y+u)]|\} \leqq \sup _{f \in D_{\mathbf{s}}}\{|\mathrm{E}[f(X)]-\mathrm{E}[f(Y)]|\} \\
& =\xi_{s}(X, Y)=W_{s}\left(P_{X}, P_{Y}\right) \leqq \mathrm{E}\left[|X-Y|^{s}\right]
\end{aligned}
$$

Observe that Theorem 8 is somewhat comparable to the $V(n)$-version of Theorem 6 with $X=T_{n}, Y=Z$. However, the function class $D_{s}=C \cap \mathrm{Lip} s$ is larger than that in Theorem 6, namely $C^{r}, r \geqq 2$; the large- $O$ order is replaced by little- $。$. In this respect see also a further concretization in Theorem 9 . From another point of view thcre states a lemma of ZoLOTAREv (see [36, Lemma 2]) that

$$
\xi_{s}(X, Y) \leqq \frac{\Gamma(1+\alpha)}{\Gamma(1+s)} B_{s}(X, Y) \quad(s>0)
$$

where

$$
\dot{B_{s}}(X, \dot{Y}):=\left\{\mathrm{E}\left[|X|^{8}\right]+\mathrm{E}\left[|Y|^{s}\right]\right\}, s>0, s=r+\alpha ; r^{\prime} \in \cdot \mathbb{N}, \alpha \in(0,1], \ldots
$$

(6.2) being valid provided $j$

i) $B_{s}(X, Y)<\infty$,

ii) $\mathrm{E}\left[X^{k}\right]=\mathrm{E}\left[Y^{k}\right],(1 \leqq k \leqq r)$.

Thẹrem 9: Let $s>0, s=r+\alpha, \alpha \in(0,1], r \in \mathbb{N}$. For two sequences of independent randóm variables with $T_{n}=\sum_{i=1}^{n} \varphi(n) X_{i}$ and $Z=\sum_{i=1}^{n} \varphi(n) Z_{n i}$, for which the $X_{i}, Z_{n i}$ satisfy $(6.3)$, one has for $f \in D_{s}$.(recall Definition 6$)$,

$$
\sup _{u \in \mathbb{R}}\left\{\left|\mathrm{E}\left[f\left(T_{n}+u\right)\right]-\mathrm{E}[f(Z+u)]\right|\right\} \leqq \frac{\Gamma(1+\alpha)}{\Gamma(1+s)}|\varphi(n)|^{8} B_{s}\left(X_{i}, \check{Z}_{n i}\right) .
$$


Proof: It follows by Lemma 8, (6.2) and a further Theorem of ZoLOTaRev [36, Theorem 2], namely $\xi_{s}\left(T_{n}, Z\right) \leqq|\varphi(n)|^{8} \sum_{i=1}^{n} \xi_{s}\left(X_{i}, Z_{n i}\right)$, that

$$
\begin{aligned}
& \sup _{u \in \mathbb{R}}\left\{\left|\mathrm{E}\left[f\left(T_{n}+u\right)\right]-\mathrm{E}[f(Z+u)]\right|\right\} \leqq \xi_{s}\left(T_{n}, Z\right) \\
& \leqq|\varphi(n)|^{s} \sum_{i=1}^{n} \xi_{s}\left(X_{i}, Z_{n i}\right) \leq|\varphi(n)|^{8} \sum_{i=1}^{n} \frac{\Gamma(1+\alpha)}{\Gamma(1+s)} B_{s}\left(X_{i}^{-}, Z_{n i}\right)
\end{aligned}
$$

Remark 6: If one would replace the pseudo-moment condition (5.2) by

$$
\sum_{i=1}^{n} \mathbf{E}\left[X_{i}^{j}-Z_{n i}^{j}\right]=0 \cdot\left(\frac{\varphi(n)^{r}}{(r-1) !} M(n)\right)
$$

'it could be shown (compare [12]) that for functions $f$ from Lip ${ }^{(r-1)} \alpha:=\forall \in C ; f^{(r-1)} \in$ Lip $\left.\alpha\right\}$, $\alpha \in(0,1]$, and for possibly dependent random variables $X_{i}$ there holds

$$
\sup _{u \in \mathbb{R}}\left\{\mathrm{E}\left[f\left(T_{n}+u\right)\right]-\mathrm{E}[J(Z+u)] \|=O\left(\left\{\frac{\varphi(n)^{r}}{2(r-1) !} M(n)\right\}^{1 \frac{1-a}{r}}\right) .\right.
$$

Comparing now (6.6) with the estimate in Theorem 9 , the right-hand side of $(6.4)$ in the particular case $s=(r-1)+1$ would be equal to $\sum_{i=1}^{n} B_{r-1}\left(X_{i}, Z_{n i}\right)=M(n, r-1)$, and the function classes in both results, namely $D_{r}$ and $\operatorname{Lip}^{(r-1)} \alpha$ with $\alpha=1$, would also be equal; likewise are the orders, namely $\varphi(n)^{r} M(n, r)^{1}$ (noting $1-(1-\alpha) / r=1$ there). However, the estimate in (6.6) is more general in the sense that it does not only hold for independent random variables $X_{i}$ as in the case of Theorem 9 but also for possibly dependent ones. Further, the pseudo-moment condition (6.5) is much weaker than (6.3), namely $\mathrm{E}\left[X_{i}^{j}\right]=\mathrm{E}\left[\check{Z}_{n i}^{j}\right], 1 \leqq j \leqq r$, $i \in \dot{N}$.

- Concludingly one can say that our results of Sections $3-5$ generalize known results on the distance of random variables to the case of general limit theorems for dependent random variablès.

The authors would like to thank Dr. Dietmar Pfeifer, Heisenberg Professor, Aachen, for his critical reading of the manuscript and valuable suggestions.

\section{REFERENCES}

[1] Anastassiod, G. A.: An improved general stochastic inequality. Bull. Soc. Math. Grece (N. S.) $24(1983), 1-11$.

[2] BASU, A. K.: On the rate of convergence in the central limit theorem for dependent random variables and random vectors. J. Multivariate Anal. 10 (1980), 565-578.

[3] BAUER, H.: Wahrscheinlichkeitstheorie und Grundzïge der Maßtheorie (2. Aufl.). Berlin: De Gruyter 1974.

[4] Bergastroem, H.: On distribution functions with a limiting stable distribution function. Arkiv Mat. 2 (1952), 463-474.

[5] BolthaÚSEN, E.: On rates of convergence in a random central limit theorem and in the central limit theorem for Markoff chains. Z. Wahrsch. Verw. Gebiete 38 (1977), 279-286.

[6] Bolthausen, E.: The Berry-Esseen theorem for functionals of discrete Markov chains. $Z$. Wahrsch. Verw. Gehiete 54 (1980), 59-73.

[7] Botzer, P. L., Hahn, L., and U. Westphal: On the rate of approximation in the central limit theorem. J. Approx. Theory 13 (1975), $327-340$.

[8] Butzer, P. L., and L. HAHN: General theorems on rates of convergence in distributions of random variables. I: General limit theorems. J. Multivariate Anal. 8 (1978), $181-201$. 
[9] Butzer; P. L., and L. HAH : On Connections Between the Rates of Norm and Weak Convergence" in the Central Limit Theorem. Math. Nachr. 91 (1979), 245-251.

[10] Botzer, P. L., Hahn, L., and M. Roeckerati: General theorems on "little-o" rates of closeness of two weighted sums of independent Hilbert space valued random variables with applications. J. Multivariate.Anal. 9 (1979), 487-510.

[11] Butzer, P. L., Haris, L., and M. Roeckeratir: Central limit theorem and weak law of large numbers with rates for martingales in Banach spaces. J. Multivariate Anal. 13,(1983), $287-301$.

[12] Butzer, P. L., and H. Kirschrink: Donsker's weak invariance principle with rates for $C[0,1]$-valued, dependent random-functions. Approx. Theory Appl. $\because \mathbf{2}(1986), 55-77$.

[13] Butzer, P. L., and D. Schulz: General Random Sum Limit Theorems for Martingales with Large- $O$ Rates. $Z$. Anal. Anw. 2 (1983), 97-109.

[14] BUtzer, P. L., and D. Schulz: The random martingale central limit theorem and weak

- law of large numbers with 0 -rates. Acta Sci. Math. (Szeged) 45 (1983), $81-94$.

[15] BuTther, P. L., and D. Schulz: Approximation theorems for martingale difference arrays with applications to randomly stopped sums. In: Math. structures - computational math. - math. modelling 2: Papers dedicated to L. Hliev's 70th anniversary (Ed.: Bl. Sendov). Sofia: Bulg. Akad. Sci. 1984, pp. $121-130$.

[16] Chusa, K. L.: Lectures from Markov Processes to Brownian Motion. New York: SpringerVerlag 1982.

[17] Dupley, R. M.: Probabilities and Metrics: convergence of laws on metric spaces, with a view to statistical testing (Lect. Notes Series: No. 45). Aarhus: Aarhus Univ. 1976.

[18] Gaenssler, P., and W. Stute: Wahrscheinlichkeitstheorie. Berlin: Springer-Verlag 1977.

[19] Ginman, I. I., and A. V. SковоноD: The Theory of Stochastic Processes II. Berlin: Springer-Verlag 1975.

[20] Gudynas, P.: Refinements of the central limit theorem for a homogeneous Markov chain. Lith. Math. J. 22 (1982), 36-45.

[21] Като, J.: Convergence rates in the central limit theorem for martingale differences. Bull. Math. Stat. 18 (1979), 1-9.

[22]'Kemeny, J. G., and J. L. Snell: Finite Markov Chains. New York: Springer-Verlag 1976.

[23] LАнА, R. G., and V. K. Rohatgi: Probability Theory. New York: John Wileý \& Sons 1979.

[24] Landers, D., and L. RogGe: On the rate of convergence in the central limit theorem for Markov chains. Z. Wahrsch.' Verw. Gebiete 35 (1976), 57-63.

[25] Lirshits, B. A.: On a central limit theorem for sums of random variables connected in a Markov chain. Dokl. Akad. Nauk SSSR 219 (1974), 797-799.

[26] Lifsuits, B. A.: On the central limit theorem for Markov chains. Theory Probab. Appl. $\mathbf{2 3}(1978), 279-296$.

[27] Lirsuits, B. A.: Invariance principle for weakly dependent variables. Theory Probab. A ppl. 29 (1985), 33-40.

[28] NAGAEv, S. V.: Some limit for stationary Markov chains. Theory Probab. Appl. 2 (1957), $378 \div 406$.

[29] Nagaev, S. V.: Möre exact statements of limit theorems for homogeneous Markov chains. Theory Probab. Appl. 6 (1961), 62-81.

[30] PadiTz, L.: Uber die Annäherung von Summenverteilungsfunktionen gegen unbegrenzt $\checkmark$ teilbare Verteilungsfunktionen in der Terminologie der Pseudomomente. Wiss. Z. Techn. Univ. Dresden 27 (1978), 1129-1133.

[31] Prakasa RaO, B. L. S.: On central limit theorems, invariance principle and rates of convergence for back ward martingale arrays. Litovsk. Mat. Sb. 19 (1979) 4, 153-165, 212.

[32] Rychlik, Z.: The order of approximation in the random central limit theorem. Lect. Notes Math. 65(; (1978), 225-236.

[33] Sazonov, V. V., and V. V. Ulyaxov: On the accuracy of normal approximation. J./Multivariate Anal. 12 (1982), $371-384$.

[34] Scotr, D. J.: Central limit theorems for martingales and for processes with stationary increments using a Skohorod representation approach. Adv. Appl. Probab. $\Xi$ (1973), $119-137$. 
[35] Strobed, J.: Könvergenzraten in zentralen Grenzwertsätzen für Martingale. Dissertation. Bochum: Univ. Bochum 1978.

[36] Zolotarev, V. M.: Approximation of distributions of sums of independent random variab: les with values in infinite-dimensional spaces. Theor. Probab. A ppl. @1 (1976), 721 - 737.

[37] Zolotarev, V. M.: On pseudomoments. Theor. Probab. Appl. 23 (1978), 269-278.

[38] Zolotarev, V. M.: Ideal metrics in the problems of probability theory and mathematical statistics. Austral. J. Statist. 21 (1979), 193-208.

Manuskripteingang: 28.09.1987

\section{VERFASSER:}

Prof. Dr. h. c..Paul L. Butzer und Dipl.:Math. Heribert Kirschfink Lehrstuhl A für Mathematik der Rheinisch-Westfälischen Technischen Hochschule

Templergraben 55

D.5100 Aachen 